\title{
Role of free fatty acids on insulin resistance
}

\author{
José Gustavo Vázquez-Jiménez', Adriana Roura-Guiberna², Luisa Reyna Jiménez-Mena² and \\ Jesús Alberto Olivares-Reyes² \\ Laboratory of Signal Transduction, Department of Biochemistry, Centro de Investigación y de Estudios Avanzados del Instituto Politécnico Nacional, \\ Ciudad de México, Mexico
}

\begin{abstract}
Free fatty acids are essential nutritional components and recent studies identified them as signaling molecules in various physiological processes. It has now been shown that high levels of free fatty acids, particularly saturated fatty acids, may be associated with insulin resistance in obese patients with type 2 diabetes mellitus. Insulin resistance is important in clinical since it is related to various diseases including type 2 diabetes mellitus, dyslipidemia, and abnormalities at cardiovascular level. Recent studies have proposed different molecular mechanisms by which these lipids may alter the signaling pathway of insulin. The purpose of this review is to highlight recent advances in the study of the effect of free fatty acids as modulators of insulin response.
\end{abstract}

KEY WORDS: Fatty acids. Insulin resistance. Insulin receptor. Insulin receptor substrate.

\section{Introduction}

Insulin resistance is a systemic condition where cells cease to respond to this hormone. Under this conditions, highly important metabolic functions of insulin mainly on hepatic, muscular and adipose tissues, such as glucose uptake and synthesis of glycogen, lipids and proteins, among other effects, are altered ${ }^{1,2}$. It is closely linked with metabolic syndrome, obesity and with the development of type 2 diabetes mellitus (DM2). According to the World Health Organization and the International Federation of Diabetes most recent statistics, more than 1500 million people in the world suffer from obesity or overweight, whereas diabetes affects more than 387 million, a figure that by the year 2035 will be reaching nearly 600 million if appropriate measures are not taken (http://www.who. int/en/index.html; http://www.idf.org).

To date, several factors that can induce insulin resistance have been identified, with increased free fatty acid (FFA) plasma concentration standing out, followed by an altered use of lipids by the muscle tissue $^{3}$. This phenomenon, which is common in obese patients, has been observed in clinical trials and has been corroborated in molecular investigations.

The mechanisms implicated in insulin resistance by the action of fatty acids (FA) include alterations in the kinase PI3K pathway, production and accumulation of ceramides, protein kinase C (PKC) activation, generation of reactive oxygen species (ROS), endoplasmic reticulum (ER) stress and membrane stiffening.

\section{Insulin actions}

Insulin is the body's main anabolic hormone; it controls crucial energetic functions, such as glucose, lipid and protein metabolism. One of its most important functions is the regulation of glucose homeostasis, since it enables its uptake and storage in muscle and adipose tissue, and favors its storage and inhibits its

\footnotetext{
Correspondence:

Jesús Alberto Olivares-Reyes

Avda. Instituto Politécnico Nacional, 2508

Col. San Pedro Zacatenco

C.P. 07360, Ciudad de México, México

E-mail: jolivare@cinvestav.mx
}

Date of reception: 01-07-2016

Date of acceptance: 08-08-2016

DOI://dx.doi.org/10.24875/GMM.M18000092
Gac Med Mex. 2017;153:773-783

Contents available at PubMed www.gacetamedicademexico.com 


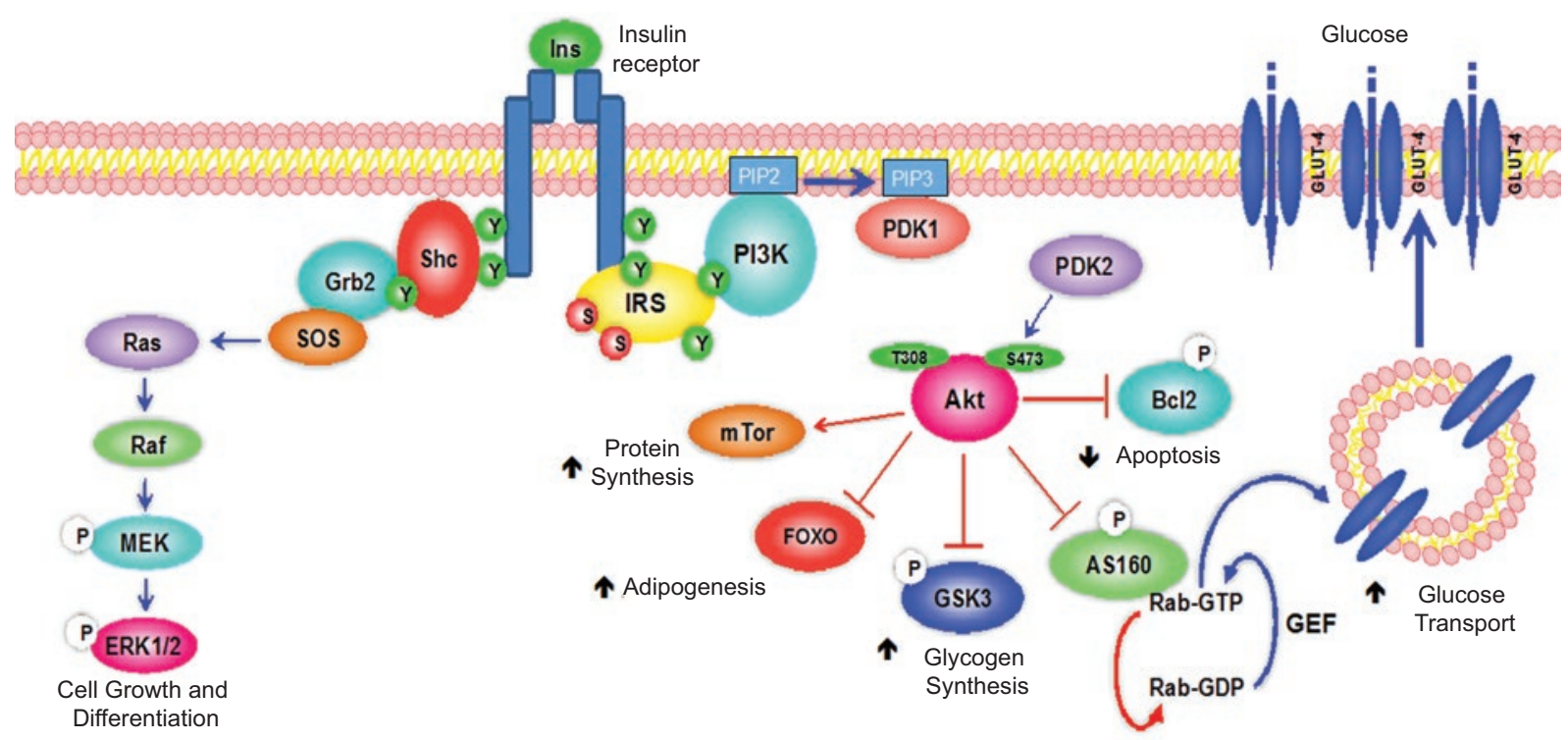

Figure 1. Insulin signaling pathway. After being activated by insulin, the receptor recruits and phosphorylates mainly two adaptor proteins: IRS and Shc. These proteins organize molecular complexes that trigger different intracellular signaling cascades. IRS-mediated pathways include $P I 3 K / A k t$, which plays a crucial role in the activation and regulation of diverse metabolic events, including glucose transport and glycogen, protein and lipid synthesis. In the case of the Shc protein, it is associated with the mitogen-activated protein kinase (MAPK) pathway activation, which regulates proliferative and growth functions (modified from Olivares-Reyes ${ }^{1}$ ). Akt: protein kinase B; AS160: 160 kDa Akt substrate; GLUT-4: glucose transporter 4; GSK3: glycogen synthase protein kinase 3; IRS: insulin receptor substrate; PDK 1: phosphoinositide-dependent protein kinase 1; PDK 2: phosphoinositide-dependent protein kinase 2; PI3K: phosphatidylinositol kinase 3; PIP2: phosphatidylinositol-4,5-biphosphate; PIP3: phosphatidylinositol-3,4,5-triphosphate.

production in the liver. In addition, insulin promotes cell division and growth by means of its mitogenic effects.

Insulin activates specific membrane surface receptors known as insulin receptors, which belong to the family of receptors with tyrosine kinase intrinsic activity. After insulin is bound, the receptor auto-phosphorylates and, in turn, phosphorylates and recruits adaptor proteins, such as the insulin receptor substrate (IRS). IRS-1 and IRS-2 are the most common substrates and intermediaries in insulin signal propagation initial stage, which organize molecular complexes and trigger intracellular signaling cascades ${ }^{4}$. Among the different PI3K pathway-activated kinases is Akt, which plays a highly important role in insulin metabolic functions, mainly in glucose uptake by muscle and adipose tissues. Akt participates in the glucose-transporting protein-4 (GLUT-4) translocation from intracellular vesicles to the cell surface in order to increase glucose uptake. Insulin also participates in gluconeogenesis inhibition and favors the synthesis of glycogen, lipids and protein in hepatic, adipose and muscle tissues, respectively ${ }^{1}$ (Fig. 1).

In turn, insulin mitogenic actions are carried out through the adaptor protein Shc, which activates the mitogen-activated kinases/Ras (MAPKs/Ras) pathway $^{1}$ (Fig. 1).

\section{Fatty acids and insulin resistance}

Insulin resistance molecular causes are diverse: it involves defects in the binding of insulin to its receptor and alterations after this bond. These alterations include a decrease in the number of insulin receptors and in kinases PI3K and Akt activity, GLUT4 transporter expression and function defects, and increased phosphorylation in serine/threonine residues of proteins such as the insulin receptor or IRS, with the latter mechanism being key in the development of insulin resistance. This can cause alterations in the association of the receptor and its substrate with other proteins, a decrease in the state of phosphorylation in both proteins' tyrosines, and a decrease in their activation and degradation ${ }^{1}$. These insulin resistance mechanisms have been associated with high FFA plasma concentrations.

FA are highly amphipathic carboxylic acids consisting of 4 to 26 carbon atom-long hydrocarbon chains, which have no branches ${ }^{5,6}$. The term FFA refers to non-esterified FAs, which stem directly from triglyceride and phospholipid metabolism. Depending on the presence or not of double bonds, they are classified in two large groups: those containing only single bonds are known as saturated fatty acids (SFA), 
where hydrogen atoms are arranged in the trans position; and FAs with double bonds, which are called unsaturated and can be found both in cis and trans configuration, although the latter rarely occurs naturally. Unsaturated FAs only containing one double bond in their structure are called monounsaturated, whereas those containing two or more are called polyunsaturated ${ }^{5,6}$.

FAs play important biological roles in living beings: they act as an important source of energy for most tissues of the body, including the heart, skeletal muscle and the liver?. In addition, they possess a wide range of biological functions, such as being a structural part of the cell membrane (being essential to its fluidity and functionality) and acting as signaling molecules $^{6}$. FAs are used as the main energy source during periods of fasting or when there is no sufficient glucose. However, when FFA values are abnormally in the body, as it occurs in obesity, a lipotoxicity state is induced, which leads to activation of different cell responses associated with this toxicity, including oxidative stress, ER stress, apoptosis and inflammation. FFAs identified as the main causative agents of these cell responses include SFAs.

The first evidence that FFA elevation plays an important role in the development of insulin resistance in muscle tissues was suggested by Randle et al. ${ }^{8}$ in 1963, based on observations that a high FFA plasma concentration is commonly associated with diabetes and other carbohydrate metabolism disorders. To date, different molecular mechanisms by means of which FFAs induce insulin resistance have been characterized, and which are next described.

\section{Fatty acid-induced insulin resistance: PKC participation}

FFAs, which often are elevated in obese individuals, play a highly important role in the association between obesity and insulin resistance ${ }^{9}$. Although the mechanisms by means of which FFAs induce insulin resistance have been partially identified, diverse evidences suggest the participation of PKC, which is activated through different mechanisms.

Currently, dietary lipid excess or obesity are known to elicit an excessive increase of these acids in the bloodstream, which exceeds the storing and oxidation capacity, causing for FFA and their metabolism intermediaries (such as linoleic acid, diacylglycerol [DAG], phosphatidic acid, lysophosphatidic acid and ceramides) to act as insulin resistance important inducers ${ }^{10}$. In particular, DAG is a potent activator of classic (PKC $\alpha$, $\mathrm{PKC} \beta \mathrm{I}, \mathrm{PKC} \beta \mathrm{II}$ and $\mathrm{PKC} \gamma)$ and new PKC isoforms $(\mathrm{PKC} \delta, \mathrm{PKC} \varepsilon, \mathrm{PKC} \eta \text { and } \mathrm{PKC} \theta)^{11}$. In contrast with PKC acute activation in response to specific stimuli, several studies have reported chronic activation of one or more PKC isoforms in cells or tissues where DAG values are elevated in the long term. Thus, PKC classic and new isoforms activated this way participate in insulin signaling negative regulation, especially by IRS-1 phosphorylation in serine residues ${ }^{12}$. Elevation of DAG values due to plasma FFA increase has been shown to promote $\mathrm{PKC} \beta \mathrm{II}$ and $\mathrm{PKC} \delta$ activation in skeletal muscle of humans. Activation of both isoforms has been associated with insulin receptor and IRS phosphorylation, which entails a decrease in the phosphorylation status in both proteins' tyrosines, thus affecting their signaling ${ }^{13}$ (Fig. 2).

In hepatic tissue of animals with a fat-rich diet, insulin resistance has been associated with an increase on DAG values that promotes the translocation of PKC $\varepsilon$ to the plasmatic membrane, where it interacts with insulin receptor catalytic domain. This interaction promotes a decrease in the receptor activity, which affects phosphorylation in IRS-2 tyrosines and Akt2 activity $^{14}$. Consequently, the capability of insulin to activate glycogen synthesis and to inhibit gluconeogenesis is altered ${ }^{15}$. Interestingly, increased DAG values and their association with $\mathrm{PKC} \varepsilon$ activation were the best insulin resistance markers in hepatic tissue of obese humans $s^{16,17}$.

On the other hand, in $\mathrm{C} 2 \mathrm{C} 12$ cells, high concentrations of palmitic acid favored DAG increase and the resulting activation of PKA $\theta$, which once activated phosphorylates IRS-1 in serine 307 , thus blocking insulin signaling ${ }^{18}$. In this same cell type, another site in IRS-1 that is phosphorylated by PKC $\theta$ was identified, serine 1101 , by arachidonic acid action ${ }^{19}$. In a recent study, Szendroedi et al. ${ }^{20}$ demonstrated that insulin resistance acute induction by lipid infusion in healthy and lean individuals is related to a transient increase in cytosolic content of DAG in skeletal muscle, which was temporally associated with $\mathrm{PKC} \theta$ activation, an increase in serine 1101 and phosphorylation inhibition in IRS-1 tyrosine, as well as insulin-induced Akt12 inactivation. Increased DAG content in muscle tissue, PKC $\theta$ activation and insulin resistance were also observed in healthy obese individuals and in obese patients with $\mathrm{DM} 2^{20}$. 


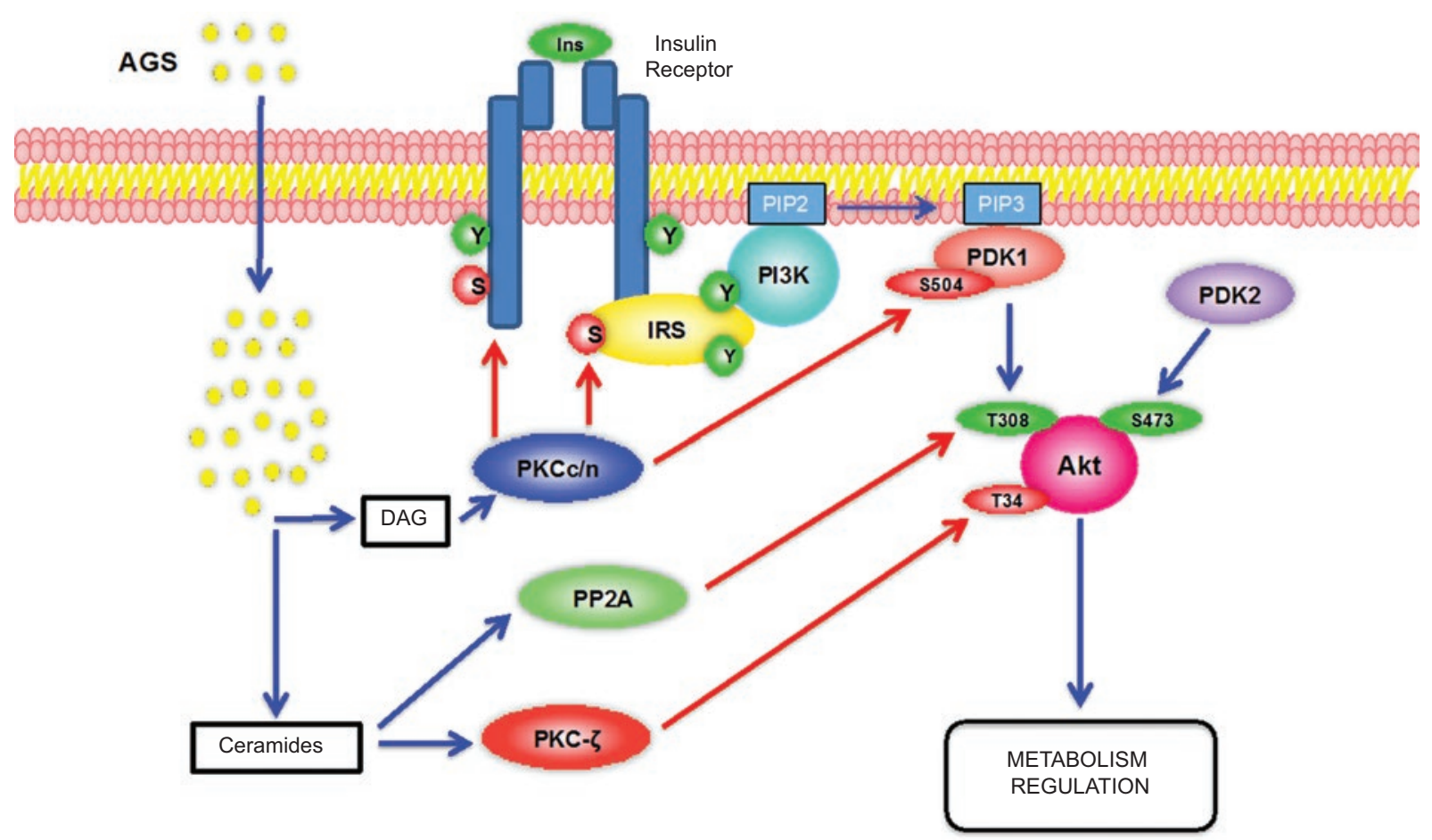

Figure 2. Diacylglycerol and ceramides as mediators of SFA-induced insulin resistance. SFA metabolism leads to the formation of intermediaries, such as DAG and ceramides. DAG is a potent activator of classic and new PKC, which participate in insulin signaling negative regulation, phosphorylating the receptor and IRS in serine residues. Additionally, other PKC phosphorylation targets have been identified, such as the PDK1 enzyme, altering its enzymatic activity and altering subsequent Akt activation. FAs also favor the synthesis of ceramides that activate PP2A, a phosphatase that dephosphorylates Akt at threonine 308, and PKC-, , which phosphorylates Akt at threonine 34. In both mechanisms, Akt activity is inhibited, thus altering insulin metabolic pathways regulation. SFA: saturated fatty acids; Akt: protein kinase B; DAG: diacylglycerol; Ins: insulin; IRS: insulin receptor substrate; PDK 1: phosphoinositide-dependent protein kinase 1; PDK 2: phosphoinositide-dependent protein kinase 2; PI3K: phosphatidylinositol kinase 3; PIP2: phosphatidylinositol-4,5-biphosphate; PIP3: phosphatidylinositol-3,4,5-triphosphate; PKCc: protein kinase, classic; PKCn: protein kinase, new; PP2A: protein phosphatase 2A; S: serine; T: threonine; Y: tyrosine.

On the other hand, Pereira et al. ${ }^{21}$ demonstrated in vivo that, in hepatic cells, FFA-induced insulin resistance is developed by oxidative stress, which is generated by NADPH oxidase (NOX) through a PKC $\delta$-dependent mechanism. Oxidative stress leads to I0B (IKK $\beta$ ) and JNK kinases activation, which mediate in IRS-1 and IRS-2 serines, with the resulting alteration of insulin signaling ${ }^{21}$ (Fig. 3).

From the results obtained by several investigation groups, it is suggested that the main regulation target in insulin signaling by PKC is IRS. However, in a study carried out by Wang et al. ${ }^{22}$, palmitic acid, through PKC $\theta$ activation, induces PDK1 phosphorylation in serine 504/505, which alters its enzymatic activity and affects subsequent Akt activation (Fig. 2). In addition, FAs were shown to attenuate insulin receptor transcriptional regulator (HGMA1) activity through a mechanism that involves $\mathrm{PKC} \varepsilon$ activation by palmitoylation. This mechanism reduces the receptor expression and, significantly, sensitivity to insulin ${ }^{23}$.

\section{Ceramides and insulin resistance}

Other intermediaries of FA metabolism involved in insulin resistance pathogenesis are ceramides, which can accumulate in cells by two main routes: cell membrane sphingomyelin hydrolysis, by action of the sphingomyelinase enzyme ${ }^{24,25}$, or by its de novo synthesis from long chain SFAs, such as palmitic acid, which implies a multiple-step biosynthetic route that occurs in the $\mathrm{ER}^{24,26}$. So far, different factors, such as tumor necrosis factor alpha (TNF- $\alpha$ ), endotoxins and diverse stress stimuli, have been found to activate sphingomyelinase, which leads to the generation of ceramides $^{24,25}$.

Ceramides' accumulation, mainly associated with palmitic acid increased concentration, has been shown to be able to affect insulin-induced Akt2 activation. This appears to be due to a direct effect on Akt2 activation, rather than by interfering in the signaling of proteins that are activated before this kinase ${ }^{27,28}$. Firstly, ceramides have been described to 


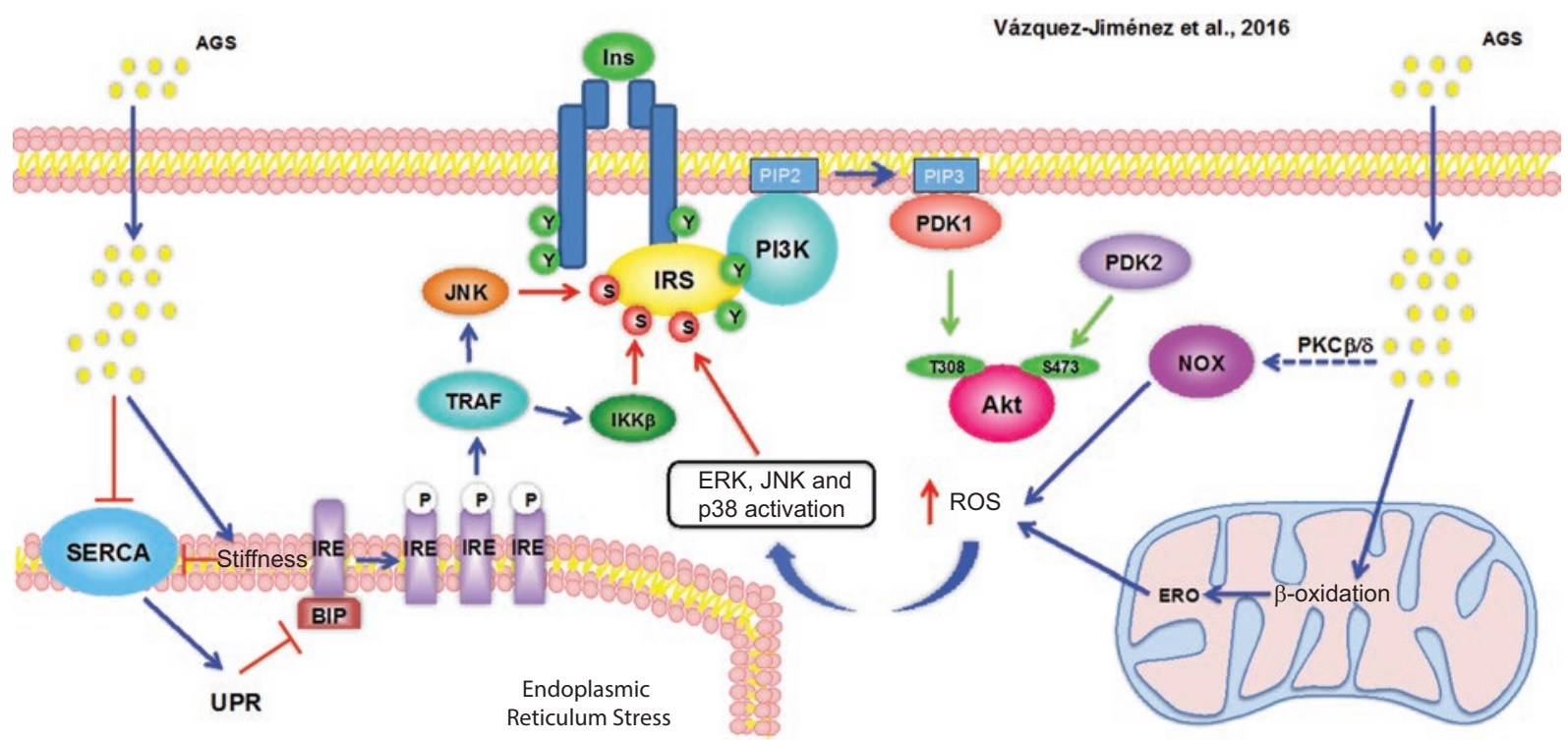

Figure 3. SFA-induced insulin resistance through reticulum stress and oxidative stress. SFAs elicit an increase in oxidative stress status by an increase in $\beta$-oxidation. In addition, SFAs can activate PKC 3 , which induces NOX activation and ROS production. Once established, oxidative stress activates multiple serine kinases, such as JNK, ERK and p38, which phosphorylate IRS. On the other hand, SFAs also promote ER stress. One of the proposed mechanisms suggests that membrane fluidity decrease by SFA-induced membrane lipid synthesis, affects the SERCA pump activity and $\mathrm{Ca}^{2+}$ concentrations, leading to reticulum stress. This condition triggers UPR, which is involved in IRE-1 activation and, through its interaction with TRAF2, drives to kinases IKK 3 and JNK activation, which in turn phosphorylate IRS. IRS phosphorylation in serine residues has been associated with a decrease in their phosphorylation status in tyrosines, which reduces their capability to interact with other signaling proteins, with the PI3K/Akt being mainly altered (modified from Vázquez-Jiménez JG et al. graphic abstract ${ }^{71}$ ). Akt: protein kinase B; BIP: binding immunoglobulin protein; ERK2/2: extracellular signal-regulated protein kinases 2/2; Ins: insulin; IKKB: IK $\beta$ kinase; inositol-requiring endonuclease; IRS: insulin receptor substrate; JNK: c-Jun amino-terminal kinase; p38: 38 kDa mitogen-activated protein kinase; PDK1: phosphoinositide-dependent protein kinase 1; PDK2: phosphoinositide-dependent protein kinase 2; PI3K: phosphatidylinositol kinase 3; PIP2: phosphatidylinositol-4,5-biphosphate; PIP3: phosphatidylinositol-3,4,5-triphosphate; ROS: reactive oxygen species; S: serine; SERCA: sarco/ endoplasmic reticulum Ca2+ ATPase; SFA: saturated fatty acid; T: threonine; TRAF: TNF- $\alpha$ receptor-associated factor; UPR: unfolded protein response $Y$ : tyrosine.

promote protein phosphatase 2 (PP2A) activation. PP2A is a seine/threonine cytoplasmic phosphatase that is expressed ubiquitously, and plays and important role in the regulation of diverse cellular processes, including metabolic enzymes, hormone receptors, kinase cascades and cell growth regulation. Under physiological conditions, insulin inhibits PP2a. PP2A has been shown to dephosphorylate Akt threonine 308 , which is one of the two residues that, together with serine 473 , are phosphorylated when the enzyme is active ${ }^{29}$ (Fig. 2). In addition, ceramides prevent Akt recruitment by activating atypical $\square \mathrm{PKC}(\mathrm{PKC})$, which phosphorylates threonine 34 that is found in Akt $\mathrm{PH}$ domain, thus preventing its binding to $\mathrm{PIP}_{3}$ and inhibiting its translocation and subsequent activation in response to insulin ${ }^{30,31}$ (Fig. 2).

Currently, there is controversy about the role played by ceramides in insulin resistance, owing to differences in the obtained results: in some studies, a direct correlation has been found between ceramides accumulation and insulin resistance, but others suggest that SFAs promote insulin resistance by an increase in DAG concentrations, with no significant changes in the ceramides content ${ }^{32,33}$.

\section{Fatty acids, oxidative stress and insulin resistance}

Oxidative stress can be defined as a state of unbalance between the production and elimination of ROS, whose overproduction by an increase in FFA plasma concentrations promotes an oxidative stress state that has been associated with mitochondrial damage and with different pathophysiological processes, such as insulin resistance, obesity and diabetes ${ }^{34}$. In the case of obesity, FFAs released by adipose tissue that has been damaged as a consequence of its expansion and of the development of hypoxia and cell death, are metabolized by muscle and hepatic tissues, where ROS overproduction and oxidative stress development has been identified ${ }^{35}$. In healthy subjects, FFA infusion causes oxidative stress and insulin resistance increase that can be reverted with antioxidants ${ }^{36}$. In turn, FFA increase in obesity elicits an increase in the 
oxidative stress state through an increase in $\beta$-oxidation of long-chain acyl-coenzyme A esters, FFAs metabolically-active form ${ }^{37}$ (Fig. 3). In addition, it has been described that, in aortic smooth muscle cells and in endothelial cells, FFAs such as palmitic acid can also stimulate ROS production through NOX activation, which promotes oxidative stress by a PKC $\beta$-dependent mechanism ${ }^{38,39}$ (Fig 3). It has been suggested that PLC induces NOX activation by $\mathrm{p} 47^{\text {phox }}, \mathrm{p} 67^{\text {phox }}$ and Rac subunits phosphorylation, thus causing their translocation to the Nox2/p22phox heterodimer, which finally entails the formation of NOX active complex ${ }^{38,39}$.

ROS production and oxidative stress activate multiple stress-sensitive signaling pathways, where kinase members of the MAPK family, such as KNK, ERK and p38. These kinases promote insulin receptor and IRS phosphorylation, which is associated with a reduction of their tyrosine phosphorylation state and with an attenuation of the insulin signal ${ }^{40}$ (Fig. 3). Nakamura et al. ${ }^{41}$ demonstrated that, in the H4IIEC3 hepatic-origin cell line, palmitic acid inhibits the effect of insulin on IRS-2 and Akt tyrosine phosphorylation, through a mechanism that involves JNK activation. They found that JNK activation is associated with an increase in palmitic acid-generated $\beta$-oxidation, which causes an excess in respiratory chain electron flow and an increased generation of mitochondrion-produced ROS. In addition, increased oxidative phosphorylation was associated with an increase in carnitine palmitoyltransferase 1a expression, which is limiting in mitochondrial FA $\beta$-oxidation ${ }^{41}$.

Oxidative stress has also been shown to stimulate transcriptional factors activation, such as nuclear factor kappa G (NFaB), activator protein 1 (AP-1) and hypoxia inducible factor 1 (HIF-1), which promote pro-inflammatory gene expression ${ }^{40}$. Furukawa et al. ${ }^{42}$ demonstrated that fat accumulation in humans and mice with obesity is correlated with the presence of systemic oxidative stress. These authors observed that, in obese mice adipose tissue, the production of ROS is increased, and that this is associated with an increase in NOX expression and a decrease in antioxidant enzymes expression. Furthermore, in cultured adipose cells, FAs stimulate NOX activity and promote an increase in adipocytokines, such as plasminogen-activator inhibitor-1, interleukin 6, TNF- $\alpha$ and monocyte chemotactic protein-1. On one hand, these pro-inflammatory cytokines contribute to obesity-associated local and generalized inflammation state and, on the other, directly induce insulin resistance $^{1,43}$
It should be observed that, in a FFA-excess chronic state, there is pancreatic beta cell dysfunction. In this context, prolonged exposure of the latter to FFA causes, on one hand, an increase in basal insulin release values and, on the other, an inhibition of glucose-induced secretion ${ }^{44}$. In addition, FFAs inhibit insulin gene expression and induce apoptosis in these cells ${ }^{45,46}$. Alteration in beta cell functions has been suggested to likely be due to ROS production and to oxidative stress state, both in animal models and in studies carried out in humans ${ }^{47-49}$.

\section{The role of fatty acids in endoplasmic reticulum stress}

New evidences establish that ER dysfunction contributes to the development of metabolic conditions, such as obesity and DM2. ER plays a determinant role in $\mathrm{Ca}^{2+}$ homeostasis and participates in membrane and secretion proteins maturation and expression. In cell stress conditions that increase ER demand and entail an overload of its functional capacity, a series of alterations known as "endoplasmic reticulum stress" is generated, including a decrease in the transportation of proteins to the Golgi apparatus, unfolding protein expression and calcium depletion of this reservoir ${ }^{50}$. Under these conditions, the ER activates a compensatory mechanism called "unfolding protein response" (UFR), which tries to reestablish ER functions homeostasis ${ }^{50}$. In case this response is insufficient, apoptosis is then unleashed ${ }^{51}$.

UPR triggers three stress sensor enzymes' activation: 1) double stranded RNA-activated protein kinase-like endoplasmic reticulum kinase (PERK); 2) inositol-requiring endoribonuclease enzyme-1 (IRE1); and 3) transcription factor 6 precursor (ATF6). Under normal conditions, these proteins are associated, through their luminal domain, with chaperon BIP/ GRP78 (binding immunoglobulin protein/78 kDa glucose-regulated protein), thus remaining inactive. Under stress conditions, BIP/GRP78 is separated from sensor enzymes to promote correct protein folding. In addition to BIP separation, all three sensor proteins are activated, which favor the expression of proteins that help with the overload of proteins and enzymes associated with protein degradation in the reticulum ${ }^{51,52}$.

On the other hand, IRE-1 activation induces its interaction with TRAF protein, a mechaniusm that favors IKK $\beta$ and JNK kinases activation, which in turn can phosphorylate IRS1 in serine residues, thus blocking 
insulin signaling ${ }^{52}$ (Fig. 3). In response to the conversion of excessive amounts of FFA and other nutrients in fats, adipose cells can develop signs of ER stress (Fig. 3). In vitro studies in adipose ${ }^{53,54}$, hepatic ${ }^{55}$ and beta pancreatic ${ }^{56}$ cell cultures have demonstrated that FFAs produce ER stress and UPR. On the other hand, an increase in FFA by a fat-rich diet or by lipid infusion elicits ER stress in adipose and hepatic tissues of rodents $^{54.57}$. While ER stress produces insulin resistance mainly through JNK activation, chaperon OPR150 overexpression in diabetic mice $(\mathrm{db} / \mathrm{db})$, or pre-treatment with chemical chaperons were demonstrated to decrease JNK activation, which suggests that the protection of cells against ER stress decreases the state of insulin resistance ${ }^{51,58-60}$.

According to the above, the JNK kinase gas emerged as a key regulator of the metabolic alterations in insulin sensitivity. JNK activity has been detected to be elevated in animal models of obesity ${ }^{58,60}$, and JNK isoforms 1 and 2 deletion protects mice from insulin resistance induced by a fat-rich diet ${ }^{61,62}$. In molecular research, experimental evidence indicates that JNK phosphorylates on one hand serine 307 IRS-1, a phosphorylation that has been associated with a decrease in insulin actions by altering the association between the insulin receptor and IRS-163,64, and on the other, it phosphorylates IKK $\beta$, which leads to NFaB activation and to inflammatory responses regulation ${ }^{50,65}$.

In addition to JNK-mediated phosphorylation, there are other mechanisms associated with ER stress-associated insulin resistance. One that can be important and relevant is protein tyrosine phosphatase-1B (PT$\mathrm{P} 1 \mathrm{~B})$ activation. This enzyme, which is attached to the ER cytosolic face membrane surface by the carboxyl-terminal extreme, acts as a negative regulator of insulin and IRS-1 actions ${ }^{66}$. Recently, a fat-rich diet was shown to induce the expression of UPR-marker proteins, as well as that of PTP1B in skeletal muscle, an increase that was prevented by treatment with the chaperone TUDCA (tauroursodeoxicholic acid), which reduces ER stress ${ }^{66}$. Controversially, Obanda and Cefalu $^{67}$ demonstrated that PTP1B expression increase by FFA per se plays no direct role in insulin resistance. They propose that the increase in ceramide values by FFA metabolism in muscle cells is associated with and altered Akt activation, which negatively regulates PTP1B activity by phosphorylation in serine 50 , thus decreasing this residue phosphorylation and increasing PTP1B activity, which promotes insulin resistance ${ }^{67}$.
On the other hand, alterations in sarco/endoplasmic reticulum $\mathrm{Ca}^{2+}$ ATPase (SERCA) expression, which have calcium elimination from cytosol and returning it to the ER as their function, are associated with ER stress and subsequently with insulin resistance. Under conditions that reduce the SERCA pump activity, a luminal calcium environment that entails reticulum chaperons' low activity is produced ${ }^{68}$. In 2010, Park et al. ${ }^{69}$ reported an important reduction of SER$\mathrm{CA}$ expression in hepatic tissues of obese mice (ob/ ob) in comparison with control mice; restoration of the pump in this tissue is associated with a decrease in RE stress, an increase in glucose tolerance and a decrease in blood glucose ${ }^{69}$. On the other hand, palmitic acid promotes ER stress in hepatic cells, with SERCA activity and ER $\mathrm{Ca}^{2+}$ concentrations being drastically altered and leading to cell death ${ }^{70}$. Recently, we demonstrated that palmitic acid induces insulin resistance in endothelial-origin cells through a biphasic mechanism, which involves an initial increase followed by a sustained reduction of SERCA protein values. Notwithstanding, palmitic acid produced a sustained inhibition of the ATPase activity of the pump. Interestingly, insulin resistance appeared before there was a SERCA expression reduction. PERK and JNK kinases activation by palmitic acid suggests that the mechanism by means of which the acid alters insulin signaling involves ER stress. SERCA overexpression reverted the palmitic acid effect on insulin resistance, indicating that the reduction of its expression and activity would be the reason why palmitic acid induces a state of resistance in endothelial cells ${ }^{71}$.

The treatment of diabetic people with rosiglitazone, an antidiabetic drug, increased SERCA expression, thus restoring the pump expression reduction observed in diabetic patients with altered hyperglyce$\mathrm{mia}^{72}$. The use of the chemical chaperon TUDCA restores SERCA pump expression and activity in obese mice $(o b / o b)^{73}$. This way, the decrease in SER$\mathrm{CA}$ expression promotes the development of ER stress, with JNK ensuing activation, which desensitizes insulin signal, thus generating a state of insulin resistance and contributing to chronic metabolic deterioration.

\section{Membrane stiffness and insulin resistance}

Different reports have demonstrated that dietary FA types determine the type of FA available for the composition of cell membranes. Membranes containing phospholipids synthesized from FFA possess a 
different and less fluid structure than those incorporating polyunsaturated (or essential) FA ${ }^{74,75}$. Membrane fluidity degree, as determined by the ratio of polyunsaturated FAs and SFAs, influences on insulin-dependent glucose and GLUT4 transporters uptake and in insulin binding to its receptor effectiveness ${ }^{75-77}$. Different experimental studies concur in pointing out that the composition of FA in muscle cell membrane phospholipids importantly influences on insulin sensitivity ${ }^{75,77,78}$.

Beyond cell membrane alterations generated by FFA uptake by phospholipids, changes in the ER and mitochondrial membranes lipid composition that affect these organelles' function have also been observed. In the case of the ER, membrane fluidity is highly important to its fusogenic activity and plasticity. Borradfaile et al. ${ }^{79}$ demonstrated that incubation with FFA causes ER stress, an effect that was associated with a higher proportion of triglycerides with FFA in their membranes. in comparison with triglycerides in control cells, and this may be associated with a decrease in ER membrane fluidity. On the other hand, cholesterol pathophysiological concentrations have been shown to decrease membrane fluidity, as well as SERCA pump activity, an effect not occurring when there are high FA concentrations present in the ER membrane. These data suggest that, if ER membrane fluidity is decreased, reticulum stress will eventually develop, which in turn might generate a state of insulin resistance $^{80}$ (Fig. 3).

In the mitochondrial membrane, FA composition and phospholipid saturation degree are important to its fluidity, its permeability and, therefore, to its appropriate finction ${ }^{81}$. Consumption of a fat rich diet has been suggested to be able to influence on the mitochondrial membrane composition, which has been associated with alterations that entail insulin resistance and DM2 $2^{82,83}$. However, recent data by Hoeks et al. ${ }^{84}$ suggest that, although in mice with a fat-rich diet changes are promoted in the mitochondrial membrane lipid composition, no alterations are observed in mitochondrial functions, such as FA oxidation, in spite of insulin resistance being induced. In fact, other studies have suggested that mitochondrial capacity in skeletal muscle is found to be increased in rodents on a fat-rich $\operatorname{diet}^{85-87}$. These findings underscore the need for further studies clarifying the importance of mitochondrial membrane composition in mitochondrial functions and its association with the development of insulin resistance.

\section{Toll-like receptor-mediated insulin resistance}

Increases in the glucose, FFA and pro-inflammatory cytokines secretion values in DM2 have been shown to have important implications for the immune system ${ }^{88,89}$. Studies in animal models and in humans have suggested a close link between DM2 and changes in innate immune system response ${ }^{90}$. In this sense, Tolllike receptors (TLR), a family of transmembrane receptors that belong to the innate immune system and that recognize pathogen-associated molecular patterns, have an important participation in the pathogenesis of insulin resistance, inflammation and DM2 ${ }^{88,89,91}$. In particular, TLR-4 have elevated expression and signaling in rodents and humans with obesity and insulin resistance, especially in insulin target tissues ${ }^{91-93}$. Although the mechanisms by means of which TLR-4 signaling is increased have not been fully defined. A possible explanation is that lipopolysaccharide values, TLR-4 natural ligand, are elevated in individuals with obesity and DM2 ${ }^{94,95}$. Interestingly, FFAs have been reported to be TLR-4 agonists as well, which suggests a possible role of these receptors in obesity-induced chronic inflammation $^{96}$. TLR-4-mediated signaling activation promotes the generation of pro-inflammatory cytokines through upregulation of several transcriptional factors, such as NF-aB and AP-197 (Fig. 4). This way, TLR-4 deficiency in $\mathrm{KO}$ mice improves insulin sensitivity and significantly attenuates inflammation in states of diet-induced obesity ${ }^{93,98,99}$. Recently, Jia et al. ${ }^{100}$ demonstrated that hepatic TLR-4-defficient mice ( $\mathrm{TI} / \mathrm{K} \mathrm{L}^{\mathrm{k}}{ }^{\mathrm{O}}$ ) show an increase in insulin sensitivity and an improvement in glucose tolerance and hepatic steatosis, in spite of the development of obesity due to a fat-rich diet. Furthermore, these KO mice showed an inflammatory response decrease in adipose tissue and in circulating inflammatory markers ${ }^{100}$. These data reinforce the idea that the immune system plays a crucial role in the development of insulin resistance and DM2.

\section{Conclusions}

Circulating FFA concentrations increase, particularly saturated FA, in pathologic conditions such as obesity, induces insulin resistance through different mechanisms described in the present review. It is otherwise interesting that, beyond the classic mechanisms identified in insulin resistance, such as IRS serine residues phosphorylation by action of different kinases such as 


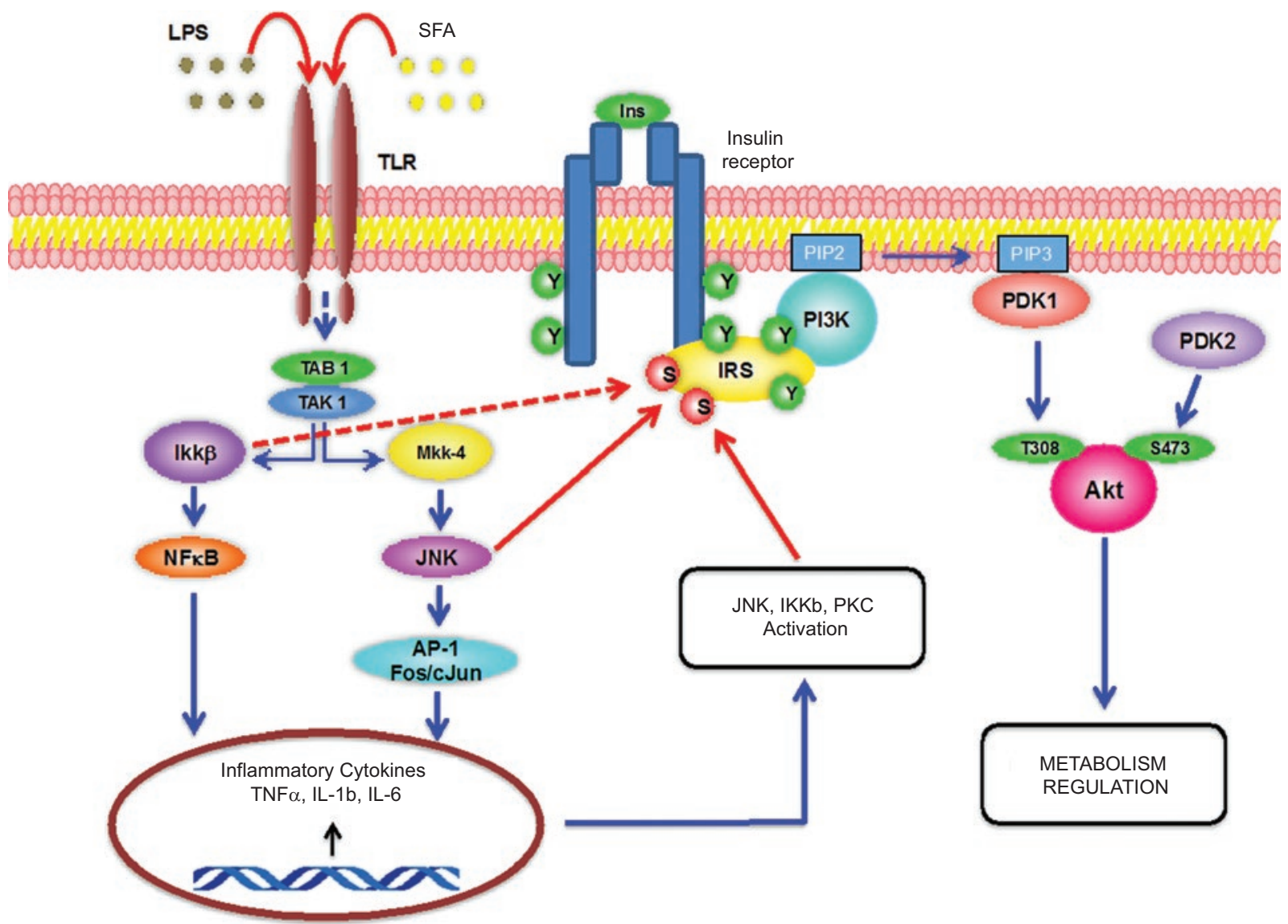

Figure 4. TLR-mediated fatty acids effects. TLR activation by LPS or SFA leads to TAK1 kinase association with TAB1 protein, a complex that promotes KKB and JNK activation, which causes IRS phosphorylation and increases the transcription of inflammatory genes that contribute to insulin resistance, through the activation of transcriptional genes such as NFKB and AP-1 (modified from Vázquez-Jiménez JG et al. ${ }^{71}$ ). Akt: protein kinase $B ; A p-1$ : activator protein 1 formed by Fos/cJun; Fos: proto-oncogene encoded by the fos gene in humans; Ins: insulin; IKK interleukin 1B; IL-6: interleukin 6; IRS: insulin receptor substrate; JNK: c-JUN amino-terminal kinase; c-JUN: c-jun gene-encoded proto-oncogene; LPS: lipopolysaccharides; MKK4: mitogen-activated protein kinase 4;; NFKB: nuclear factor kappa B; PDK1: phosphoinositide-dependent protein kinase 1; PDK2: phosphoinositide-dependent protein kinase 2; PI3K: phosphatidylinositol kinase 3; PIP2: phosphatidylinositol-4,5-biphosphate; PIP3: phosphatidylinositol-3,4,5-triphosphate; PKC: protein kinase C; S: serine; SFA: saturated fatty acid; T: threonine; TAK1: transforming growth factor beta 1-activated protein kinase 1; taB1: TAK1-binding protein; TLT: Toll-like receptors; TNF- $\alpha$; tumor necrosis factor alpha; Y: tyrosine.

PKC, KNK and IKK $\beta$, recent evidence suggests that SFAs can regulate proteins that are activated downstream to IRS, at the level of PDK1 and Akt, as well as inflammatory cytokines expression. This indicates the complexity of the mechanisms involved in insulin resistance, the complexity of the mechanisms involved in insulin resistance, the multiplicity of regulation spots and why is it so difficult to establish therapeutic measures; however, adequate study and understanding of such mechanisms can provide a better view on them and broaden the therapeutic routes in the face of this disorder.

\section{Acknowledgements}

JGVJ was a CONACYT grant holder (No. 233232). ARG is a CONACYT grant holder (No. 245147). This work was supported by the SEP/CONACYT sectoral fund (Code 167673). We also thank Diego Olivares Hernández for his review and suggestions to improve the writing of the manuscript.

\section{Conflicts of interests}

The authors declare not having any conflicts of interests.

\section{References}

1. Olivares-Reyes JA. Bases moleculares del síndrome metabólico y resistencia a la insulina. En: Garibay-Nieto S, editor. Obesidad en la edad pediátrica: prevención y tratamiento. Mexico, D.F.: Corinter; 2012. p. 185-214.

2. Kahn SE, Hull RL, Utzschneider KM. Mechanisms linking obesity to insulin resistance and type 2 diabetes. Nature. 2006;444:840-6.

3. Karpe F, Dickmann JR, Frayn KN. Fatty acids, obesity, and insulin resistance: time for a reevaluation. Diabetes. 2011;60:2441-9.

4. Jensen $M$, De Meyts $P$. Molecular mechanisms of differential intracellular signaling from the insulin receptor. Vitam Horm. 2009;80:51-75. 
5. Nelson DL, Cox MM. Lehninger principles of biochemistry. $6^{\text {th }}$ ed. New York, USA: MacMillan; 2013.

6. Legrand-Poels S, Esser N, L'homme L, et al. Free fatty acids as modulators of the NLRP3 inflammasome in obesity/type 2 diabetes. Biochem Pharmacol. 2014:92:131-41.

7. Rachek LI. Free fatty acids and skeletal muscle insulin resistance. Prog Mol Biol Transl Sci. 2014;121:267-92.

8. Randle PJ, Garland PB, Hales CN, et al. The glucose fatty-acid cycle its role in insulin sensitivity and the metabolic disturbances of diabetes mellitus. Lancet. 1963;281:785-9.

9. Lewis GF, Carpentier A, Adeli K, et al. Disordered fat storage and mobilization in the pathogenesis of insulin resistance and type 2 diabetes. Endocr Rev. 2002;23:201-29.

10. Capurso C, Capurso A. From excess adiposity to insulin resistance: the role of free fatty acids. Vascul Pharmacol. 2012;57:91-7.

11. Farese RV. Protein kinase C. En: LeRoith D, Taylor SI, Olefsky JM, editores. Diabetes mellitus: a fundamental and clinical text. $3^{\text {rd }}$ ed. Philadelphia, USA: Lippincott; 2000. p. 301-16.

12. Schmitz-Peiffer $C$. The tail wagging the dog - regulation of lipid metabolism by protein kinase C. FEBS J. 2013;280:5371-83.

13. Youngren J. Regulation of insulin receptor function. Cell Mol Life Sci. 2007;64:873-91.

14. Samuel VT, Liu ZX, Wang A, et al. Inhibition of protein kinase $C$ epsilon prevents hepatic insulin resistance in nonalcoholic fatty liver disease. J Clin Invest. 2007;117:739-45.

15. Samuel VT, Liu ZX, Qu X, et al. Mechanism of hepatic insulin resistance in non-alcoholic fatty liver disease. J Biol Chem. 2004;279:32345-53.

16. Kumashiro N, Erion DM, Zhang D, et al. Cellular mechanism of insulin resistance in nonalcoholic fatty liver disease. Proc Natl Acad Sci U S A 2011;108:16381-5.

17. Perry RJ, Samuel VT, Petersen KF, et al. The role of hepatic lipids in hepatic insulin resistance and type 2 diabetes. Nature. 2014;510:84-91.

18. Coll $T$, Eyre $E$, Rodríguez-Calvo $R$, et al. Oleate reverses palmitate-induced insulin resistance and inflammation in skeletal muscle cells. J Biol Chem. 2008;283:11107-16.

19. Li Y, Soos TJ, Li X, et al. Protein kinase $C \theta$ inhibits insulin signaling by phosphorylating IRS1 at Ser1101. J Biol Chem. 2004;279:45304-7.

20. Szendroedi J, Yoshimura T, Phielix E, et al. Role of diacylglycerol activation of $\mathrm{PKC} \theta$ in lipid-induced muscle insulin resistance in humans. Proc Natl Acad Sci. 2014:111:9597-602.

21. Pereira S, Park E, Mori Y, et al. FFA-induced hepatic insulin resistance in vivo is mediated by $\mathrm{PKC} \delta$, NADPH oxidase, and oxidative stress. Am J Physiol Endocrinol Metab. 2014;307:E34-E46.

22. Wang C, Liu M, Riojas RA, et al. Protein kinase C theta (PKC theta)-dependent phosphorylation of PDK1 at Ser504 and Ser532 contributes to palmitate-induced insulin resistance. J Biol Chem. 2009/284:2038-44

23. Dasgupta S, Bhattacharya S, Maitra S, et al. Mechanism of lipid induced insulin resistance: activated PKC epsilon is a key regulator. Biochim Biophys Acta. 2011;1812:495-506.

24. Kolesnick RN, Kronke M. Regulation of ceramide production and apoptosis. Annu Rev Physiol. 1998;60:643-65.

25. Lipina C, Hundal HS. Sphingolipids: agents provocateurs in the pathogenesis of insulin resistance. Diabetologia. 2011:54:1596-607.

26. Yang G, Badeanlou L, Bielawski J, et al. Central role of ceramide biosynthesis in body weight regulation, energy metabolism, and the metabolic syndrome. Am J Physiol Endocrinol Metab. 2009;297:E211-24.

27. Schmitz-Peiffer C, Craig DL, Biden TJ. Ceramide generation is sufficient to account for the inhibition of the insulin-stimulated PKB pathway in C2C12 skeletal muscle cells pretreated with palmitate. J Biol Chem. 1999;274:24202-10.

28. Stratford S, Hoehn KL, Liu F, et al. Regulation of insulin action by ceramide: dual mechanisms linking ceramide accumulation to the inhibition of Akt/protein kinase B. J Biol Chem. 2004;279:36608-15.

29. Resjö S, Göransson O, Härndahl L, et al. Protein phosphatase $2 A$ is the main phosphatase involved in the regulation of protein kinase $B$ in rat adipocytes. Cell Signal. 2002;14:231-8.

30. Powell DJ, Hajduch E, Kular G, et al. Ceramide disables 3-phosphoinositide binding to the pleckstrin homology domain of protein kinase $B(P K B) /$ Akt by a PKCzeta-dependent mechanism. Mol Cell Biol. 2003;23 7794-808.

31. Fox TE, Houck KL, O'Neill SM, et al. Ceramide recruits and activates protein kinase $\mathrm{C} \zeta(\mathrm{PKC} \zeta)$ within structured membrane microdomains. J Biol Chem. 2007;282:12450-7.

32. Aerts JM, Boot RG, van Eijk M, et al. Glycosphingolipids and insulin resistance. Adv Exp Med Biol. 2011;721:99-119.

33. Lee JS, Pinnamaneni SK, Eo SJ, et al. Saturated, but not n-6 polyunsaturated, fatty acids induce insulin resistance: role of intramuscular accumulation of lipid metabolites. J Appl Physiol. 2006;100:1467-74.

34. Henriksen EJ, Diamond-Stanic MK, Marchionne EM. Oxidative stress and the etiology of insulin resistance and type 2 diabetes. Free Radic Bio Med. 2011:51:993-9.

35. Schenk S, Saberi M, Olefsky JM. Insulin sensitivity: modulation by nutrients and inflammation. J Clin Invest. 2008;118:2992-3002.
36. Paolisso G, Giugliano D. Oxidative stress and insulin action: is there a relationship? Diabetologia. 1996;39:357-63.

37. Wojtczak L, Schonfeld P. Effect of fatty acids on energy coupling processes in mitochondria. Biochim Biophys Acta. 1993;1183:41-57.

38. Inoguchi $T$, Li $P$, Umeda $F$, et al. High glucose level and free fatty acid stimulate reactive oxygen species production through protein kinase C-dependent activation of $\mathrm{NAD}(\mathrm{P}) \mathrm{H}$ oxidase in cultured vascular cells. Diabetes. 2000;49:1939-45.

39. Chinen I, Shimabukuro M, Yamakawa K, et al. Vascular lipotoxicity: endothelial dysfunction via fatty-acid-induced reactive oxygen species overproduction in obese Zucker diabetic fatty rats. Endocrinology. 2007:148:160-5

40. Rains JL, Jain SK. Oxidative stress, insulin signaling, and diabetes. Free Radic Biol Med. 2011;50:567-75.

41. Nakamura S, Takamura T, Matsuzawa-Nagata N, et al. Palmitate induces insulin resistance in H4IIEC3 hepatocytes through reactive oxygen species produced by mitochondria. J Biol Chem. 2009;284:14809-18.

42. Furukawa S, Fujita T, Shimabukuro M, et al. Increased oxidative stress in obesity and its impact on metabolic syndrome. J Clin Invest. 2004:114:1752-61.

43. Soumaya K. Molecular mechanisms of insulin resistance in diabetes. En: Ahmad S, editor. Diabetes. New York: Springer; 2013. p. 240-51.

44. Jacqueminet $S$, Briaud I, Rouault $C$, et al. Inhibition of insulin gene expression by long-term exposure of pancreatic beta cells to palmitate is dependent on the presence of a stimulatory glucose concentration. Metabolism. 2000;49:532-6.

45. Shimabukuro M, Zhou YT, Levi M, et al. Fatty acid-induced beta cell apoptosis: a link between obesity and diabetes. Proc Natl Acad Sci U S A. 1998;95:2498-502.

46. Kaneto $\mathrm{H}$, Nakatani $\mathrm{Y}$, Kawamori $\mathrm{D}$, et al. Role of oxidative stress, endoplasmic reticulum stress, and $\mathrm{c}$-Jun $\mathrm{N}$-terminal kinase in pancreatic beta-cell dysfunction and insulin resistance. Int $\mathrm{J}$ Biochem Cell Biol. 2006;38:782-93.

47. Zhang $X, B a o Y, K e ~ L$, et al. Elevated circulating free fatty acids levels causing pancreatic islet cell dysfunction through oxidative stress. J Endocrinol Invest. 2010;33:388-94.

48. Xiao C, Giacca A, Lewis GF. Oral taurine but not N-acetylcysteine ameliorates NEFA-induced impairment in insulin sensitivity and beta cell function in obese and overweight, non-diabetic men. Diabetologia. 2008; 51:139-46.

49. Oprescu Al, Bikopoulos G, Naassan A, et al. Free fatty acid-induced reduction in glucose-stimulated insulin secretion: evidence for a role of oxidative stress in vitro and in vivo. Diabetes. 2007;56:2927-37.

50. Guerrero-Hernández A, León-Aparicio D, Chávez-Reyes J, et al. Endoplasmic reticulum stress in insulin resistance and diabetes. Cell Calcium. 2014;56:311-22

51. Marciniak SJ, Ron D. Endoplasmic reticulum stress signaling in disease. Physiol Rev. 2006;86:1133-49.

52. Salvado L, Palomer X, Barroso E, et al. Targeting endoplasmic reticulum stress in insulin resistance. Trends Endocrinol Metab. 2015;26:438-48.

53. Guo W, Wong S, Xie W, et al. Palmitate modulates intracellular signaling, induces endoplasmic reticulum stress, and causes apoptosis in mouse 3T3-L1 and rat primary preadipocytes. Am J Physiol Endocrinol Metab. 2007;293:E576-86

54. Kawasaki N, Asada R, Saito A, et al. Obesity-induced endoplasmic reticulum stress causes chronic inflammation in adipose tissue. Sci Rep. 2012;2:799.

55. Wei $Y$, Wang D, Topczewski F, et al. Saturated fatty acids induce endoplasmic reticulum stress and apoptosis independently of ceramide in liver cells. Am J Physiol Endocrinol Metab. 2006;291:E275-81.

56. Karaskov $E$, Scott $C$, Zhang $L$, et al. Chronic palmitate but not oleate exposure induces endoplasmic reticulum stress, which may contribute to INS-1 pancreatic $\beta$-cell apoptosis. Endocrinology. 2006;147:3398-407.

57. Boden G. Obesity, insulin resistance and free fatty acids. Curr Opin Endocrinol Diabetes Obes. 2011:18:139-43.

58. Ozcan U, Cao Q, Yilmaz E, et al. Endoplasmic reticulum stress links obesity, insulin action, and type 2 diabetes. Science. 2004;306:457-61.

59. Nakatani $\mathrm{Y}$, Kaneto $\mathrm{H}$, Kawamori D, et al. Involvement of endoplasmic reticulum stress in insulin resistance and diabetes. J Biol Chem. 2005;280:847-51.

60. Ozcan U, Yilmaz E, Ozcan L, et al. Chemical chaperones reduce ER stress and restore glucose homeostasis in a mouse model of type 2 diabetes. Science. 2006;313:1137-40.

61. Hirosumi J, Tuncman G, Chang L, et al. A central role for JNK in obesity and insulin resistance. Nature. 2002;420:333-6.

62. Tuncman G, Hirosumi J, Solinas G, et al. Functional in vivo interactions between JNK1 and JNK2 isoforms in obesity and insulin resistance. Proc Natl Acad Sci U S A. 2006;103:10741-6.

63. Aguirre V, Werner ED, Giraud J, et al. Phosphorylation of Ser307 in insulin receptor substrate-1 blocks interactions with the insulin receptor and inhibits insulin action. J Biol Chem. 2002;277:1531-7.

64. Hotamisligil GS, Peraldi $P$, Budavari $A$, et al. IRS-1-mediated inhibition of insulin receptor tyrosine kinase activity in TNF-alpha- and obesity-induced insulin resistance. Science. 1996;271:665-8. 
65. Bakker W, Eringa EC, Sipkema $P$, et al. Endothelial dysfunction and diabetes: roles of hyperglycemia, impaired insulin signaling and obesity. Cell Tissue Res. 2009;335:165-89.

66. Panzhinskiy $E$, Hua $Y$, Culver $B$, et al. Endoplasmic reticulum stress upregulates protein tyrosine phosphatase $1 \mathrm{~B}$ and impairs glucose uptake in cultured myotubes. Diabetologia. 2013;56:598-607.

67. Obanda DN, Cefalu WT. Modulation of cellular insulin signaling and PTP1B effects by lipid metabolites in skeletal muscle cells. J Nutr Biochem. 2013;24:1529-37.

68. Caspersen C, Pedersen PS, Treiman M. The sarco/endoplasmic reticulum calcium-ATPase $2 b$ is an endoplasmic reticulum stress-inducible protein. J Biol Chem. 2000;275:22363-72.

69. Park SW, Zhou Y, Lee J, et al. Sarco(endo)plasmic reticulum Ca2+-ATPase $2 b$ is a major regulator of endoplasmic reticulum stress and glucose homeostasis in obesity. Proc Natl Acad Sci U S A. 2010;107:19320-5.

70. Zhang J, Li Y, Jiang S, et al. Enhanced endoplasmic reticulum SERCA activity by overexpression of hepatic stimulator substance gene prevents hepatic cells from ER stress-induced apoptosis. Am J Physiol Cell Physiol. 2014;306:C279-90.

71. Vázquez-Jiménez JG, Chávez-Reyes J, Romero-García T, et al. Palmitic acid but not palmitoleic acid induces insulin resistance in a human endothelial cell line by decreasing SERCA pump expression. Cell Signal. 2016;28:53-9

72. Randriamboavonjy V, Pistrosch F, Bolck B, et al. Platelet sarcoplasmic endoplasmic reticulum $\mathrm{Ca2+-ATPase}$ and mu-calpain activity are altered in type 2 diabetes mellitus and restored by rosiglitazone. Circulation. 2008;117:52-60.

73. Ceylan-Isik AF, Sreejayan N, Ren J. Endoplasmic reticulum chaperon tauroursodeoxycholic acid alleviates obesity-induced myocardial contractile dysfunction. J Mol Cell Cardiol. 2011;50:107-16.

74. Hussein JS. Cell membrane fatty acids and health. International Journal of Pharmacy and Pharmaceutical Sciences. 2013;5:38-46.

75. Weijers RN. Lipid composition of cell membranes and its relevance in type 2 diabetes mellitus. Curr Diabetes Rev. 2012;8:390-400.

76. Yorek M, Leeney E, Dunlap J, et al. Effect of fatty acid composition on insulin and IGF-I binding in retinoblastoma cells. Invest Ophthalmol Vis Sci. 1989;30:2087-92.

77. Grunfeld C, Baird KL, Kahn CR. Maintenance of 3T3-L1 cells in culture media containing saturated fatty acids decreases insulin binding and insulin action. Biochem Biophys Res Commun. 1981;103:219-26.

78. Borkman M, Storlien LH, Pan DA, et al. The relation between insulin sensitivity and the fatty-acid composition of skeletal-muscle phospholipids. N Engl J Med. 1993;328:238-44.

79. Borradaile NM, Han X, Harp JD, et al. Disruption of endoplasmic reticulum structure and integrity in lipotoxic cell death. J Lipid Res. 2006;47:2726-37.

80. Li Y, Ge M, Ciani L, et al. Enrichment of endoplasmic reticulum with cholesterol inhibits sarcoplasmic-endoplasmic reticulum calcium ATPase-2b activity in parallel with increased order of membrane lipids: implications for depletion of endoplasmic reticulum calcium stores and apoptosis in cholesterol-loaded macrophages. J Biol Chem. 2004;279:37030-9.

81. Haag M, Dippenaar NG. Dietary fats, fatty acids and insulin resistance: short review of a multifaceted connection. Med Sci Monit. 2005;11:RA359-67.

82. Sparks LM, Xie H, Koza RA, et al. A high-fat diet coordinately downregulates genes required for mitochondrial oxidative phosphorylation in skeletal muscle. Diabetes. 2005;54:1926-33.
83. Brehm A, Krssak M, Schmid Al, et al. Increased lipid availability impairs insulin-stimulated ATP synthesis in human skeletal muscle. Diabetes. 2006;55:136-40.

84. Hoeks J, Wilde J, Hulshof MF, et al. High fat diet-induced changes in mouse muscle mitochondrial phospholipids do not impair mitochondrial respiration despite insulin resistance. PLoS One. 2011;6:e27274.

85. Hancock $\mathrm{CR}$, Han $\mathrm{DH}$, Chen M, et al. High-fat diets cause insulin resistance despite an increase in muscle mitochondria. Proc Natl Acad Sci U S A. 2008;105:7815-20.

86. Iossa S, Mollica MP, Lionetti L, et al. Skeletal muscle oxidative capacity in rats fed high-fat diet. Int J Obes Relat Metab Disord. 2002;26:65-72.

87. Turner N, Bruce CR, Beale SM, et al. Excess lipid availability increases mitochondrial fatty acid oxidative capacity in muscle: evidence against a role for reduced fatty acid oxidation in lipid-induced insulin resistance in rodents. Diabetes. 2007;56:2085-92.

88. Dasu MR, Devaraj S, Zhao L, et al. High glucose induces toll-like receptor expression in human monocytes: mechanism of activation. Diabetes. 2008; $57: 3090-8$

89. Schwartz EA, Zhang WY, Karnik SK, et al. Nutrient modification of the innate immune response: a novel mechanism by which saturated fatty acids greatly amplify monocyte inflammation. Arterioscler Thromb Vasc Biol. 2010;30:802-8.

90. Pino SC, Kruger AJ, Bortell R. The role of innate immune pathways in type 1 diabetes pathogenesis. Curr Opin Endocrinol Diabetes Obes. 2010;17:126-30

91. Song MJ, Kim KH, Yoon JM, et al. Activation of Toll-like receptor 4 is associated with insulin resistance in adipocytes. Biochem Biophys Res Commun. 2006;346:739-45.

92. Reyna SM, Ghosh S, Tantiwong $P$, et al. Elevated toll-like receptor 4 expression and signaling in muscle from insulin-resistant subjects. Diabetes. 2008;57:2595-602.

93. Shi H, Kokoeva MV, Inouye K, et al. TLR4 links innate immunity and fatty acid-induced insulin resistance. J Clin Invest. 2006;116:3015-25.

94. Cani PD, Amar J, Iglesias MA, et al. Metabolic endotoxemia initiates obesity and insulin resistance. Diabetes. 2007;56:1761-72.

95. Liang H, Hussey SE, Sánchez-Ávila A, et al. Effect of lipopolysaccharide on inflammation and insulin action in human muscle. PLoS One. 2013;8:e63983.

96. Lee JY, Sohn KH, Rhee SH, et al. Saturated fatty acids, but not unsaturated fatty acids, induce the expression of cyclooxygenase-2 mediated through Toll-like receptor 4. J Biol Chem. 2001;276:16683-9.

97. Osborn O, Olefsky JM. The cellular and signaling networks linking the immune system and metabolism in disease. Nat Med. 2012;18: 363-74.

98. Suganami T, Mieda T, Itoh M, et al. Attenuation of obesity-induced adipose tissue inflammation in $\mathrm{C} 3 \mathrm{H} / \mathrm{HeJ}$ mice carrying a Toll-like receptor 4 mutation. Biochem Biophys Res Commun. 2007;354:45-9.

99. Poggi M, Bastelica D, Gual P, et al. $\mathrm{C} 3 \mathrm{H} / \mathrm{HeJ}$ mice carrying a Toll-like receptor 4 mutation are protected against the development of insulin resistance in white adipose tissue in response to a high-fat diet. Diabetologia. 2007;50:1267-76.

100. Jia L, Vianna CR, Fukuda M, et al. Hepatocyte Toll-like receptor 4 regulates obesity-induced inflammation and insulin resistance. Nat Commun. 2014;5:3878. 